\title{
Corona: Katalysator für Digitalisierung und Transparenz?
}

\author{
Eine Studie über die Auswirkungen der Pandemie
} Johannes Schnelle, Henning Schöpper und Wolfgang Kersten, Technische Universität Hamburg

\begin{abstract}
Die Corona-Krise hatte einen unübersehbaren Einfluss auf die Beschaffungssituation in den globalen Lieferketten, an den sich die Unternehmen schnell anpassen mussten. Die Auswirkungen verdeutlichen, dass sich die Unternehmen zur Reduzierung der Risiken mit der Struktur und der Transparenz in den Lieferketten beschäftigen müssen. Im folgenden Beitrag wird untersucht, über welche Kenntnisse die Akteure verfügen und wie sie diese durch die Digitalisierung verbessern wollen. Die Ergebnisse belegen, dass die Unternehmen bisher nur über geringe Kenntnisse jenseits ihrer direkten Lieferanten verfügen, jedoch zunehmend in der Lage sind, an benötigte Daten aus der Lieferkette zu gelangen. Zugleich zeigen die Ergebnisse, dass noch Potenzial zur Steigerung der Transparenz und der Nutzung von Daten vorhanden ist.
\end{abstract}

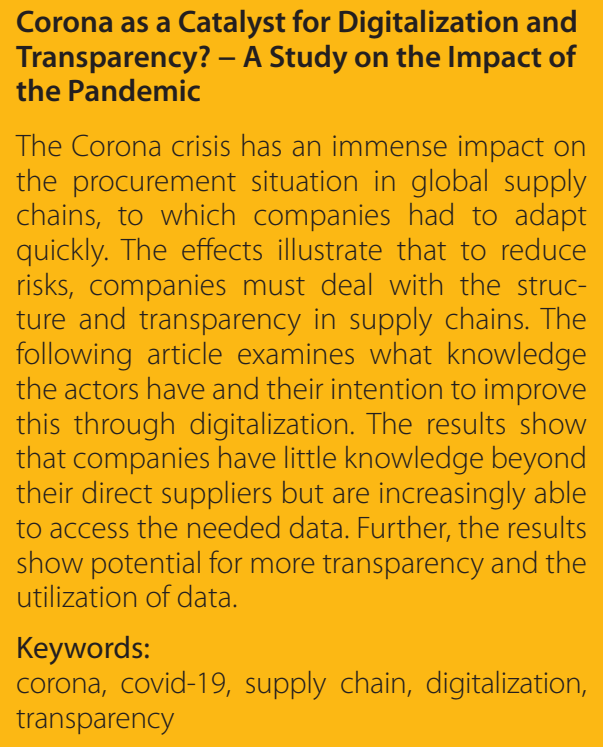

Corona as a Catalyst for Digitalization and Transparency? - A Study on the Impact of the Pandemic

The Corona crisis has an immense impact on the procurement situation in global supply chains, to which companies had to adapt quickly. The effects illustrate that to reduce risks, companies must deal with the structure and transparency in supply chains. The following article examines what knowledge the actors have and their intention to improve this through digitalization. The results show that companies have little knowledge beyond their direct suppliers but are increasingly able to access the needed data. Further, the results show potential for more transparency and the utilization of data.

Keywords: corona, covid-19, supply chain, digitalization, transparency

Neben vielfältigen sozialen und ökologischen Fragen wirkt sich die Corona-Krise wirtschaftlich vor allem im Kontext der globalen Lieferketten aus. So wurde zu Beginn der Krise im Januar mit der Schließung vieler Betriebe in China die Beschaffungsseite und der Materialfluss zahlreicher Unternehmen deutlich gestört. Im Anschluss folgten ab Mitte März negative wirtschaftliche Auswirkungen auf Europa und den Rest der Welt, wodurch zusätzlich die Nachfrageseite geschwächt wurde. Im Ergebnis sind durch die Krise die Lieferketten weltweit erheblich unter Druck geraten, wobei der Handelsstreit zwischen den USA und China und der anstehende Austritt Großbritanniens aus der Europäische Union bereits vorher zu weiteren Unsicherheiten geführt haben [1-3].

Die Krise hat die verschiedenen Branchen in Deutschland in unterschiedlichem Maße getroffen. Bspw. hatte die Automobilbranche zu Beginn der Krise vor allem mit Problemen in der Beschaffung zu kämpfen, anschließend sind die Umsätze im April 2020 um 61\% zurückgegangen [4]. Zwar konnte sich die Absatzlage wieder etwas erholen, lag aber im August 2020 noch $21 \%$ unter dem Vorkrisenniveau.

Neben der produzierenden Industrie haben die Auswirkungen auch zwangsläufig zu Einschränkungen in der Logistikbranche geführt. Zunächst machte sich dies insbesondere anhand der teilweise geschlossenen Grenzen innerhalb Europas oder des ausfallenden Warenstroms aus dem asiatischen Raum bemerkbar [5]. In Folge musste die Logistikbranche sich an die veränderten Gegebenheiten anpassen. Zum einen ist es zu einer Verlagerung oder Reduzierung der Warenströme gekommen. Eine aktuelle Studie von PWC geht bspw. davon aus, dass die Logistikleistungen im Jahr 2020 um $8,6 \%$ schrumpfen werden [6]. Zum anderen haben z. B. im Bereich der KEP-Dienstleister die B2C-Sendungen aufgrund des zunehmenden Onlinehandels zugenommen, während Sendungen an Geschäftskunden weniger geworden sind [7].

Die Krise hat dadurch deutlich gemacht, wie verwundbar die Lieferketten in ihrer globalen Ausrichtung sind. Daher wurde in den Medien

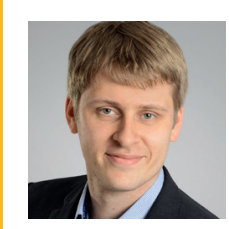

Johannes Schnelle M. Sc. arbeitet als wissenschaftlicher Mitarbeiter an der Technischen Universität Hamburg am Institut für Logistik und Unternehmensführung.

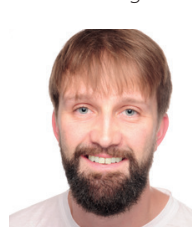

Henning Schöpper M. Sc. arbeitet als wissenschaftlicher Mitarbeiter an der Technischen Universität Hamburg am Institut für Logistik und Unternehmensführung.

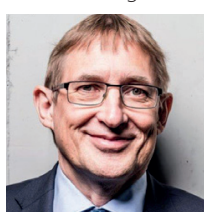

Prof. Dr. Dr. h. c. Wolfgang Kersten leitet das Institut für Logistik und Unternehmensführung an der Technischen Universität Hamburg.

www.tuhh.de/logu 
und der Wissenschaft über die kurz- und langfristigen Auswirkungen der Krise diskutiert. Zur Beschreibung der Fähigkeit von Lieferketten, widerstandsfähig gegenüber möglichen Gefahren zu sein, wird häufig der Begriff,Resilienz' genutzt $[8,9]$. Resilienz beschreibt die Fähigkeit eines Systems Störungen durch proaktive Maßnahmen zu widerstehen und den Ursprungszustand wiederherzustellen oder die Position in Folge zu verbessern [10]. Es werden verschiedene Faktoren genannt, welche die Resilienz der Lieferketten beeinflussen, wie das Wissen über die eigene Lieferkette oder akkuraten Kenntnisse über die eigenen Bestände [11]. Darüber hinaus erfordert Resilienz eine widerstandsfähige Lieferantenstruktur. Unternehmen müssen sich daher damit auseinandersetzen, aus welchen Regionen sie ihre Waren beschaffen und wie breit sie ihre Lieferantenbasis aufstellen [12]. Als weiterer zukunftsweisender Faktor wird eine engere Zusammenarbeit in der Lieferkette in Kombination mit der fortschreitenden Digitalisierung gesehen. Dies ermöglicht die notwendige Transparenz, durch die besser auf Störungen in der Lieferkette reagiert werden kann [10]. Eine entsprechende Transparenz setzt neben der technischen Infrastruktur die Bereitschaft zum Datenaustausch und damit Vertrauen zwischen den Akteuren voraus. In Summe lassen sich fünf Kernthemen identifizieren, die sich durch die Corona-Krise verändern [vgl. 13, 14]:

- Bedeutungsgewinn der Flexibilität zur Sicherung der Wettbewerbsfähigkeit

- Identifikation von Resilienz als Erfolgsfaktor für Wertschöpfungsketten

- Beschleunigung der Digitalisierung und Entwicklung digitaler Geschäftsmodelle

- Regionalisierung der Produktion

- Veränderung der Arbeitsorganisation durch Flexibilisierung

Ziel der vorliegenden Untersuchung ist es, insbesondere zu den Aspekten Transparenz und Digitalisierung einen Überblick über die aktu- elle Lage in den Lieferketten zu schaffen und einen Ausblick für zukünftige Entwicklungen zu geben.

\section{Forschungsansatz}

Um die Auswirkungen der Corona-Krise auf die Lieferketten zu untersuchen, wurde in Zusammenarbeit mit der Logistikinitiative Hamburg eine Querschnittsstudie im Raum Hamburg durchgeführt. Die Entwicklung der globalen Lieferketten ist besonders wichtig für den Logistikstandort Hamburg, da sich Veränderungen in den Lieferketten schnell auf die lokale Wirtschaft auswirken, die zu einem großen Teil von Logistik- und Handelsunternehmen geprägt ist. Die Befragung erfolgte mittels eines onlinegestützten Fragebogens, der an die Mitglieder der Logistikinitiative Hamburg versendet wurde. Im Zeitraum vom 05. bis zum 28. August 2020 wurden 150 vollständige Rückläufer erzielt. Ein Teil der Befragten stammte aus den Bereichen wie z. B. Software und IT, Beratung sowie Bildung. Da für diese Unternehmen die Auswirkungen auf die Lieferketten nur schwierig zu beurteilen sind, wurden diese in einem ersten Schritt aus der Analyse herausgenommen. Im Ergebnis resultierte eine Stichprobe von 108 Befragten, die vergleichend mittels Tableau $^{\circledast}$ einer statistischen Analyse unterzogen wurde.

Die Stichprobe (vgl. Bild 1) setzt sich überwiegend aus Logistikdienstleistern (79\%) und zu einem geringeren Umfang aus Industrie- und Handelsunternehmen (21\%) zusammen. Diese verteilen sich zu $69 \%$ auf kleine und mittlere Unternehmen (KMU) (weniger als 500 Mitarbeiter) sowie zu $31 \%$ auf Großunternehmen.

\section{Transparenz in Lieferketten}

Die Lieferketten haben sich in den vergangenen Jahrzehnten durch die Globalisierung

\section{Größe der Unternehmen}

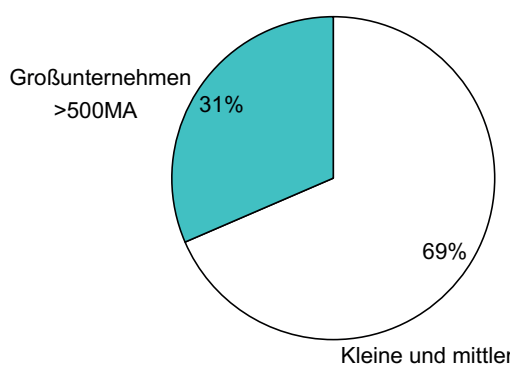

$<500 \mathrm{MA}$

\section{Branche der Unternehmen}

Handel und Verarbeitendes Gewerbe

$\mathrm{n}=108$

Bild 1: In der Befragung vertretene Unternehmensgrößen und Branchen. 


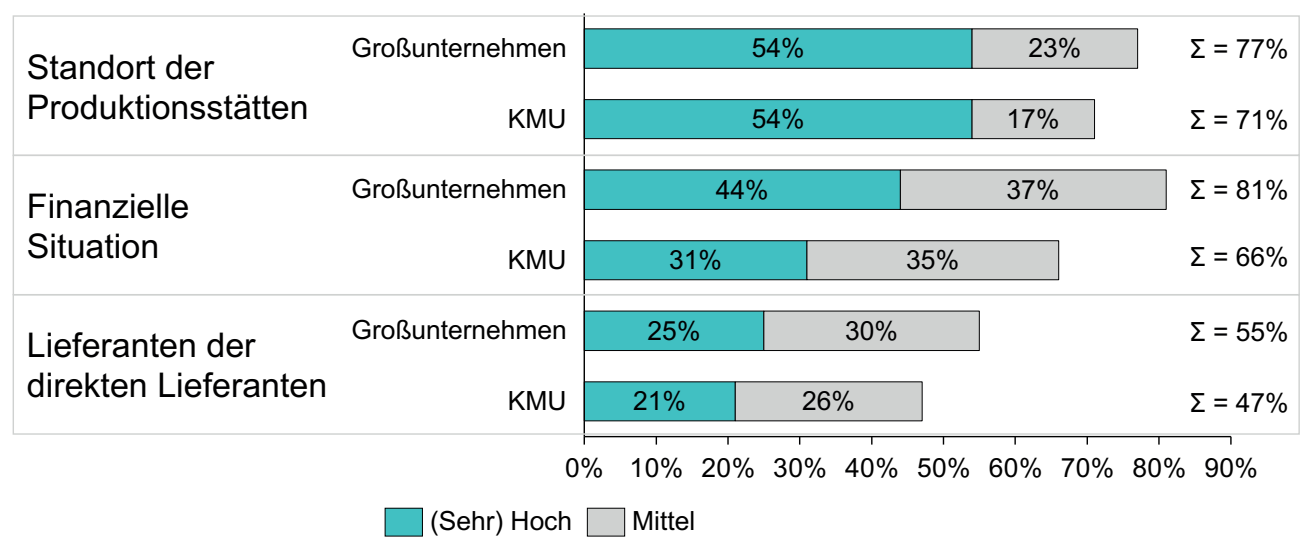

Bild 2: Anteil der Unternehmen mit mittlerem bis hohem Kenntnisstand über die Lieferkette.

deutlich verändert. Getrieben durch Kostendruck [15] und die Konzentration auf Kernkompetenzen in Kombination mit gefallenen Handelsbeschränkungen haben viele Unternehmen in der Vergangenheit Produktionskapazitäten in Niedriglohnländer verlagert („Offshoring") [16, 17].

Während durch diese Entwicklung Kosten reduziert und neue Absatzpotenziale erschlossen werden konnten, hat die Produktionsverlagerung zu neuen Herausforderungen geführt. Als Folge stehen viele Unternehmen vor der Herausforderung, die Produktqualität aufrechtzuerhalten und eine ausreichende Reaktionsfähigkeit in der Lieferkette zu gewährleisten [18]. Darüber hinaus birgt das Offshoring zusätzliche Risiken durch politische Unsicherheiten oder Naturkatastrophen. Dadurch fallen die realen Kostenersparnisse oft deutlich geringer aus als geplant. Um mit der entstehenden Komplexität und den daraus resultierenden Risiken in der Lieferkette umgehen zu können, erscheint daher ein ausreichendes Wissen über die Lieferantenstruktur notwendig [11]. Dies würde es den Unternehmen ermöglichen, drohende Risiken rechtzeitig zu erkennen und entsprechende Maßnahmen proaktiv ergreifen zu können.

Studien zeigen jedoch, dass der Kenntnisstand vieler Unternehmen über die eigene Lieferkette im Allgemeinen gering ausgeprägt ist. So können aktuell nur $8 \%$ der Unternehmen der Bekleidungsindustrie ihre Produkte bis zum Ursprung zurückverfolgen [19] und $81 \%$ der Konsumgüterhersteller besitzen keine oder nur eingeschränkte Transparenz über die Nachhaltigkeitsaktivitäten ihrer Zulieferer [20]. Diese Beispiele zeigen, dass für Unternehmen branchenübergreifend oft nur eine eingeschränkte Sichtbarkeit der stromaufwärts gelegenen Aktivitäten der Lieferkette besteht. Wei- tere Studien haben jedoch gezeigt, dass durch mehr Transparenz die Effizienz in Lieferketten erhöht und sowohl Kosten sowie Risiken reduziert werden können [21, 22]. Darüber hinaus wurden weitere negative Effekte fehlender Transparenz identifiziert, wie geringere Kostenkontrolle, mangelnder Einblick in wichtige technologische Entwicklungen und Schwierigkeiten nachhaltige und sozial verantwortliche Produktionsbedingungen bei den Zulieferern sicherzustellen [23].

\section{Ergebnisse der Befragung}

Die Ergebnisse der Befragung bestätigen weitestgehend, wie in Bild 2 dargestellt, die oben beschriebenen Beobachtungen. So geben 54\% der befragten Unternehmen an, über einen hohen Kenntnisstand bzgl. der Standorte der direkten Lieferanten zu verfügen. Bei der finanziellen Situation der direkten Lieferanten sind die Unternehmen bereits weniger gut informiert, hier geben $44 \%$ der Befragten aus Großunternehmen an, über hohe bis sehr hohe Kenntnisse zu verfügen. Auffällig ist, dass KMU hier mit 31\% insgesamt deutlich weniger Unternehmen hohe Kenntnisse vorweisen können. Allerdings verfügen über die beiden Unternehmenskategorien hinweg zumindest zwei Drittel über einen mittleren bis hohen Kenntnisstand.

Ein anderes Bild ergibt sich bei dem Kenntnisstand über die direkten Lieferanten hinaus. Hier zeigt sich, dass nur $25 \%$ der Großunternehmen bzw. 21\% der KMU hohe bzw. sehr hohe Kenntnisse vorweisen. Selbst unter Einbeziehung der Unternehmen mit mittlerem Kenntnisstand kann man davon ausgehen, dass nur rund die Hälfte der Unternehmen über einen über die direkten Lieferanten hinausgehenden Kenntnisstand verfügt.
Literatur

[1] Handfield, R. B.; Graham, G.i Burns, L.: Corona virus, tariffs, trade wars and supply chain evolutionary design, IJOPM ahead-of-print, 2020.

[2] Klös, H.-P.: Nach dem Corona-Schock: Digitalisierungspotenziale für Deutschland, 2020, IW-Policy Paper 14/2020.

[3] Raehlmann, I.: Corona! Die Krise der Verschlankung und ihre Folgen (ger), Zeitschrift fur Arbeitswissenschaft, 2020, S. 1-6.

[4] Kraftfahrtbundesamt: Fahrzeugzulassungen im September 2020: Pressemitteilung Nr. 23/2020. URL: https:// www.kba.de/SharedDocs/ Pressemitteilungen/DE/2020/ pm_23_2020_fahrzeugzulassungen_09_2020_pdf.pdf? blob=publicationfile $\& v=4$ Abrufdatum: 24.11.2020.

[5] Ankel, S.: Trucks are forming 37-mile-long queues at European borders after authorities started closing them to stop the coronavirus spread. URL https://www.businessinsider.com/coronavirus-europe-borders-shut-trucks-traffic-jam-2020-3? $r=D E \& \mid R=T$ Abrufdatum: 11.11.2020,

[6] Bauer, I. u.a.: Transport and logistics barometer: 2020 mid-year analysis of $M \& A$ deals, joint ventures and strategic alliances in the transport and logistics industry. URL: https://www.pwc.de/de/ transport-und-logistik/transport-and-logistics-barometer-h1-2020.pdf.

[7] BIEK: Rekord-Weihnachten: 420 Millionen Sendungen an Privatpersonen erwartet. URL: https://www.biek.de/presse/ meldung/rekord-weihnachten-420-millionen-sendungen-an-privatpersonen-erwartet.html Abrufdatum: 22.11.2020.

[8] Ponomarov, S. Y.; Holcomb, M. C.: Understanding the concept of supply chain resilience, International Journal of Logistics Management, Jg. 20, Nr. 1, 2009, S. 124-143. 
[9] van Hoek, R.: Research opportunities for a more resilient post-COVID-19 supply chain - closing the gap between research findings and industry practice, IJOPM, Jg. 40, Nr. 4, 2020, S. 341-355.

[10] Scholten, K.; Schilder, S.: The role of collaboration in supply chain resilience, Supply Chain Management, Jg. 20, Nr. 4, 2015, S. 471-484.

[11] Wilding, R., Dohrmann, K. und Wheatly, M.: Post-Coronavirus Supply Chain Recovery: The Journey Towards the New Normal. URL: https://www. dhl.com/content/dam/dhl/ global/core/documents/pdf/ glo-core-post-covid-emewhite-paper.pdf Abrufdatum: 20.07.2020.

[12] Roscoe, S.; Skipworth, H.; Aktas, E.; Habib, F.: Managing supply chain uncertainty arising from geopolitical disruptions: evidence from the pharmaceutical industry and brexit, IJOPM, ahead-of-print, 2020.

[13] Hippold, S.: 6 Strategies for a More Resilient Supply Chain. URL: https://www.gartner. com/smarterwithgart ner/6-strategies-for-a-more-resilient-supply-chain/ $\mathrm{Ab}$ rufdatum: 03.09.2020.

[14] Falk, S. u.a.: Corona und die Folgen: Zehn Thesen zur Zukunft digitaler Geschäftsmo-delle für Industrie 4.0 in der Post-Corona-Ökonomie. URL: $\quad$ https://www.plattform-i40.de/PI40/Redaktion/ DE/Downloads/Publikati on/Corona_Thesen.pdf? blob=publicationFile \&v $=\overline{5}$ Abrufdatum: 21.09.2020

[15] Kersten, W.; See, B. von; Lodemann S.; Grotemeier, C. Trends und Strategien in Logistik und Supply Chain Management - Entwicklungen und Perspektiven einer nachhaltigen und digitalen Transformation, BVL.digital GmbH, 2020.

[16] Farrell, D.: Offshoring: Value Creation through Economic Change, Journal of Management Studies, Jg. 42, Nr. 3, 2005, S. 675-683.

[17]OECD: Offshoring and Employment: Trends and Impacts, 2007.

[18] Heim, E.; Matiz, D.; Ehrat, M. Offshoring oder Reshoring? ZWF, Jg. 109, Nr. 12, 2014, S. 920-922.
Neben dem Informationsstand über die Lieferanten zeigen die Ergebnisse der Befragung Potenziale für den verstärkten Austausch operativer Daten auf (vgl. Bild 3). So geben z. B. $61 \%$ der Befragten an, Bedarfsprognosen aus der Lieferkette zu benötigen, allerdings verfügen $25 \%$ dieser Befragten nicht über diese Daten. Ein ähnliches Bild zeigt sich bei den Daten über Materialflussstörungen. Hier geben $27 \%$ der Befragten an, nicht über die benötigten Daten zu verfügen. Über alle Datentypen hinweg zeigen die Ergebnisse ferner, dass seit der Corona-Krise der Datenaustausch gesteigert wurde. Es verbleibt allerdings ein erheblicher Anteil an Unternehmen, deren Informationsbedürfnisse bisher nicht ausreichend gedeckt werden können.

Eine häufig formulierte These ist, dass Corona die Digitalisierung vorantreibt. Deutsche Unternehmen, vor allem KMU, sehen sich seit längerem mit erheblichen Herausforderungen bei der digitalen Transformation konfrontiert [24]. In der Frage, inwiefern die Corona-Krise diese Entwicklung vorantreibt, wird jedoch selten unterschieden, ob es sich um eine Digitalisierung von Geschäftsprozessen oder um weitreichende Digitalisierungsmaßnahmen bis hin zur Entwicklung neuer Geschäftsmodelle handelt. Die Befragung liefert hierzu einige Hinweise (vgl. Bild 4). So planen die meisten Unternehmen auf Basis der Krisenerkenntnisse die Ausweitung ihrer Investitionen in die Digitalisierung. Dabei steht insbesondere die Digitalisierung von Geschäftsprozessen im Vordergrund, in die knapp 77 \% sowohl der KMU als auch der Großunternehmen verstärkt investieren wollen. Im Bereich Künstliche Intelligenz zeigt sich, dass hier der Anteil der Großunternehmen, die einen Investitionsbedarf sehen, deutlich größer ist. So wollen knapp 51 \% der Befragten aus Großunternehmen ihre Investi- tionen in Künstliche Intelligenz erhöhen, während der Anteil mit $35 \%$ bei KMU deutlich geringer ausfällt. Hingegen sehen mit $63 \%$ weit mehr KMU Investitionspotenziale in der Entwicklung neuer Geschäftsmodelle als Großunternehmen (49\%). Diese Entwicklung zeigt auf, dass die Unternehmen nicht nur daran interessiert sind, ihre bisherigen Prozesse krisenfester und zukunftsorientierter zu gestalten, sondern sich auch strategisch an die neuen Gegebenheiten anpassen.

\section{Fazit}

Die Ergebnisse der Studie belegen insgesamt, dass sich durch die Corona-Krise das Bewusstsein der Unternehmen über die eigene Lieferkette verändert. So zeigt die Studie, dass der Kenntnisstand über die Lieferkette - insbesondere über die direkten Lieferanten hinaus - im Allgemeinen bisher eher gering ausgeprägt ist und mehr Transparenz in der Lieferkette notwendig ist.

Ein wichtiger Baustein zum Schaffen von Transparenz in der Lieferkette ist die Bereitschaft zum Teilen von Daten. Die Studie zeigt, dass die Hamburger Unternehmen in Folge der Krise Daten von ihren Partnern aus der Lieferkette erhalten. Es verbleibt allerdings noch ein erheblicher Anteil der Befragten, deren Datenbedürfnisse bisher nicht umfassend gedeckt werden können. Dabei fehlen den Unternehmen vor allem die sensiblen Informationen über Bedarfsprognosen und Materialflussstörungen der Partner, welche eine hohe Relevanz für die kurz- und mittelfristige Anpassung der Ressourcen haben. Das Ergebnis der Umfrage bleibt zwar die Antwort schuldig, ob dies an fehlenden Informationen bei den Partnern selbst oder dem Unwillen, diese zu teilen, liegt. Die Auswirkungen der Corona-Krise haben al-

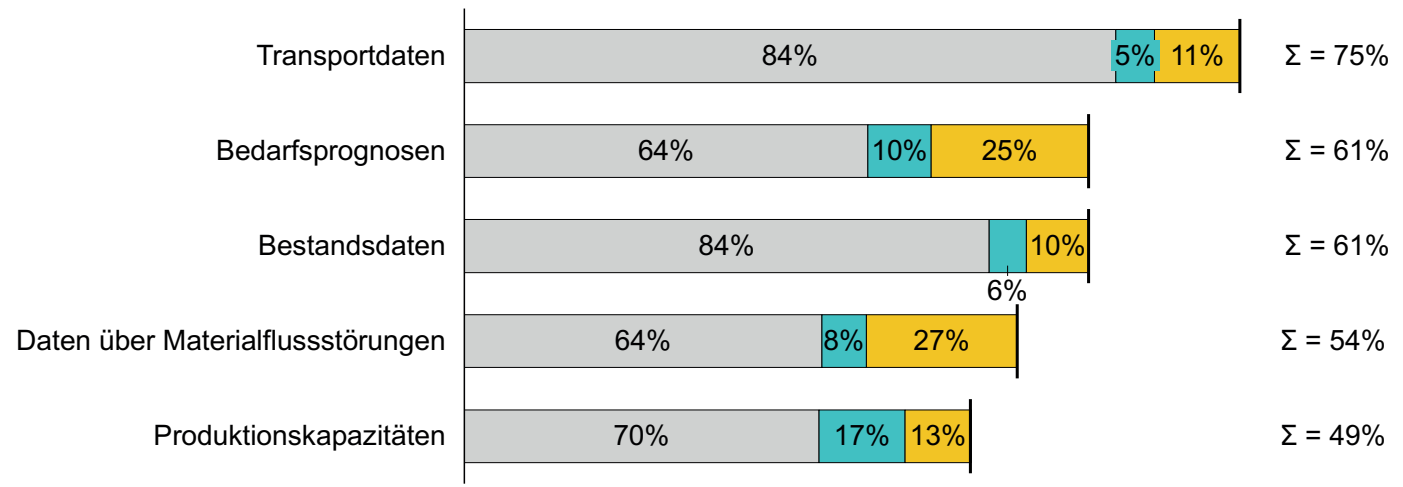

Vor der Krise verfügbar $\square$ Seit der Krise verfügbar $\square$ Nicht verfügbar

Bild 3: Anteil der befragten Unternehmen mit Datenbedarf aus der Lieferkette. 


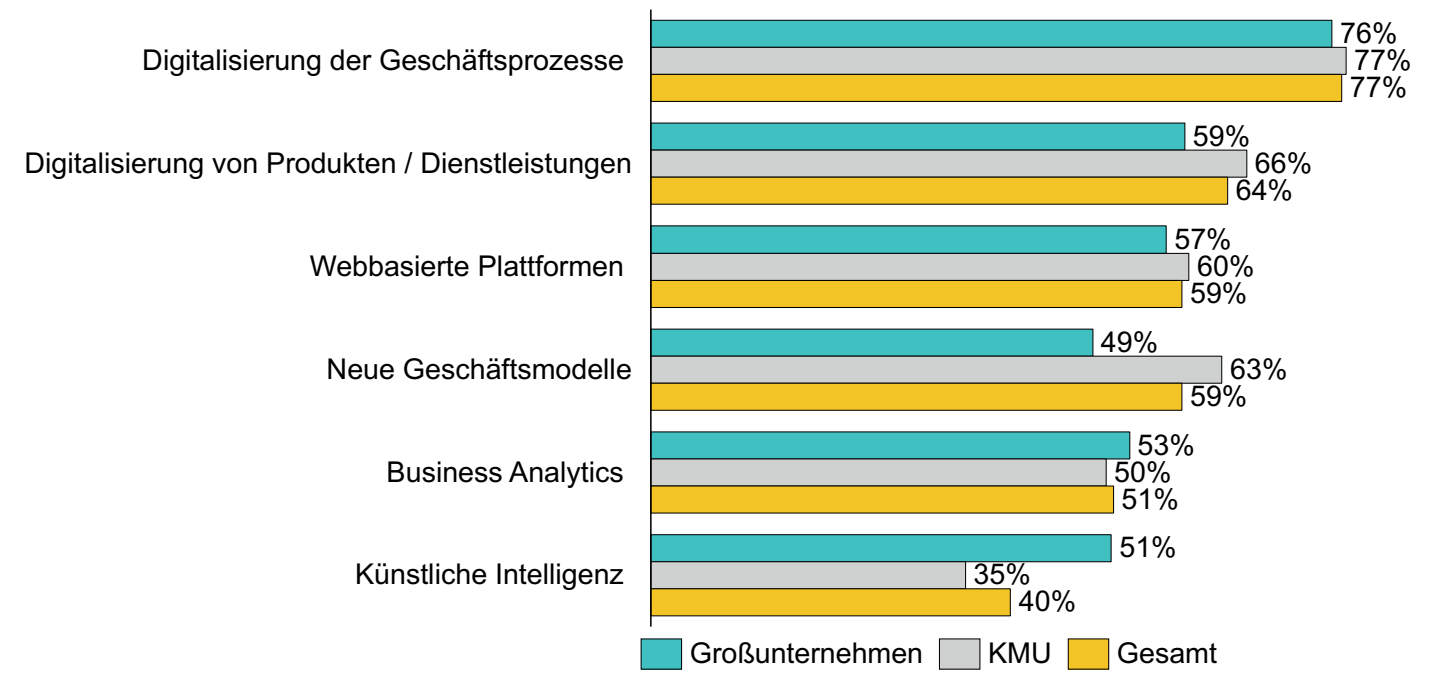

Bild 4: Anteil der Unternehmen, die ihre Investitionen in die Digitalisierung erhöhen wollen.

lerdings deutlich gemacht, dass für eine hohe Reaktionsfähigkeit eine guten Datenlage über die eigene Lieferkette erforderlich ist. Daraus lässt sich ableiten, dass weiterer Handlungsbedarf zum vertrauensvollen Austausch der Daten besteht.

Rückschlüsse auf die Fragestellung, wie Daten zukünftig geteilt werden können, bieten die Ergebnisse zu den Investitionen in die Digitalisierung. Die Ergebnisse belegen, dass das Bewusstsein für die notwendige Digitalisierung in den Unternehmen gestiegen ist. Insbesondere die verstärkten Investitionen in die Digitalisierung von Geschäftsprozessen und Produkten bzw. Dienstleistungen lassen darauf schließen, dass hier von den Unternehmen weiterer Handlungsbedarf gesehen wird. Es bleibt allerdings zum Teil offen, wie die Unternehmen es schaffen werden, die entstehenden Datenmengen gewinnbringend für die Entscheidungsfindung zu nutzen. Zwar geben rund 59 \% sowohl der Großunternehmen als auch der KMU an, zunehmend in webbasierte Plattformen investieren zu wollen, wodurch die Daten internen Akteuren als auch externen Partnern zugänglich gemacht werden können. Es folgen allerdings bei den KMU mit einem deutlichen Abstand Unternehmen, die ihre Investitionen in Business Analytics erhöhen wollen (50\%) und mit einem noch erheblich größeren Abstand solche, die in Künstliche Intelligenz investieren wollen (35\%). Hingegen ist die Differenz zwischen Investitionen in die Nutzung von Daten und der Bereitstellung von Daten bei Großunternehmen geringer ausgeprägt. Dies lässt tendenziell darauf schließen, dass Großunternehmen mit der Digitalisierung ihrer Prozesse schon weiter vorangeschritten sind. Somit können für die Großunternehmen Investitionen in die Nutzung der Daten stärker in den Vordergrund rücken. Es sollte aber auch den KMU bewusst sein, dass sie die Vorteile der Digitalisierung erst dann vollständig ausschöpfen können, wenn sie ihre Datenbasis in die Entscheidungsfindung einfließen lassen.

Die Ergebnisse der Studie zeigen somit, dass die Corona-Krise als ein Katalysator für die Umsetzung bestehender Trends wie Digitalisierung, Transparenz und unternehmensübergreifender Datenaustausch gesehen werden kann [14, 15]. Diese Entwicklung scheint nicht nur geeignet, effizientere Prozesse in den Lieferketten zu gewährleisten, sondern trägt vielmehr wesentlich zur Errichtung flexibler und widerstandsfähiger Lieferstrukturen bei.

Unternehmen sollten allerdings vermeiden, wieder in eine ähnliche Situation wie nach der Finanzkrise zu kommen. Es konnte beobachtet werden, dass die Unternehmen in Folge der Krise einen Fokus auf die Analyse der Lieferantenstruktur und den Ausbau ihres Risikomanagements gelegt haben, sich jedoch mit der Zeit wieder zunehmend mit der Effizienzsteigerung und Schaffung von Innovationen beschäftigt haben [25]. Für dauerhaft widerstandsfähige und flexible Lieferketten ist es dagegen notwendig, dass diese Entwicklungen sowohl in einem kontinuierlichen Change Management als auch im Supply Chain Risikomanagement verankert werden.

\section{Schlüsselwörter:}

Corona, COVID-19, Supply Chain, Digitalisierung, Transparenz
[19] Tatzenko, J. u. a.:The 2019 Ethical Fashion Report: The Truth Behind the Barcode. URL: https://apo.org.au/sites/default/ files/resource-files/2019-04/ apo-nid229606.pdf Abrufdatum: 24.04.2020

[20] The Sustainability Consortium: Greening Global Supply Chains: From Blind Spots To Hotspots To Action. Impact Report. URL: https://www. sustainabilityconsortium.org/ tsc-downloads/greeningglobal-supply-chains-fromblindspots-to-hotspots-to-action/?wpdmdl=19769\&ind= 1501155000091 Abrufdatum: 24.04.2020.

[21] Nooraie, S. V.: Mellat Parast, M. A Multi-Objective Approach to Supply Chain risk Management: Integrating Visibility with Supply and Demand Risk, Int J Prod Econ, Jg. 161, 2015, S. 192-200.

[22] Yu, M.-C.; Goh, M.: A Multi-Objective Approach to Supply Chain Visibility and Risk, European Journal of Operational Research, Jg. 233, Nr. 1, 2014, S. $125-130$.

[23] Choi, T. Y. Linton, T. Don't let your Supply Chain Control your Business, Harvard Business Review, Jg. 12, Nr. 89, 2011, S. 112-117.

[24] Schöpper, H.: Lodemann, S. Dörries, F.; Kersten, W.: Digitalisierung deutscher KMU im Branchenvergleich, 140M, Jg. 2018, Nr. 2, 2018, S. 38-42

[25]DVZ Redaktion: „Der ökonomische Antrieb der Globalisierung bleibt sehr stark". URL: https://www.dvz.de/rubriken/ land/detail/news/der-oekonomische-antrieb-der-globalisierung-bleibt-sehr-stark html Abrufdatum: 05.05.2020. 